\title{
How to Make Zawiya Neighborhoods Green (Debate of Walkability)
}

\author{
Huda A. Elbasha ${ }^{1,2^{*}}$, Mehmet Emre Aysu ${ }^{1}$ \\ ${ }^{1}$ Department of Architecture, Institute of Sciences, University of Istanbul Okan, Istanbul, Turkey \\ ${ }^{2}$ Higher Institutes of Science and Technology of Zawiya, Libya
}

\begin{abstract}
DOI: $\underline{10.36348 / \text { sjet.2019.v04i12.002 }}$
| Received: 06.12.2019 | Accepted: 17.12.2019 | Published: 23.12.2019
\end{abstract}

*Corresponding author: Huda A. Elbasha

\section{Abstract}

The paper discusses walkability in low-income areas of the small Libyan cities, specifically in the city of Zawiya, which borders the capital, Tripoli. The purpose of this study is to explore executive methods of how to implement the walkability of the city, as an element of urban design that is concerned with city design. The city began preparing a comprehensive development strategy after years of neglect and civil war. The sustainable ways approach is encouraged in the areas of environmental, social and economic development. The municipality pursues the development of the city center and its urban areas such as streets, squares and parks. It also aims to link streets, reduce cars, and promote pedestrians to strengthen social ties and promote culture. The researcher aims to help the municipality and draw up a plan to develop the streets and transform them into more vital places. The study is based on the spatial data analysis approach through the analysis of traditional geographic data.

Keywords: Zawiya, Libya, Walkability, Sustainable urban design, pedestrian.

Copyright @ 2019: This is an open-access article distributed under the terms of the Creative Commons Attribution license which permits unrestricted use, distribution, and reproduction in any medium for non-commercial use (NonCommercial, or CC-BY-NC) provided the original author and source are credited.

\section{INTRODUCTION}

When thinking about making this world, must first design it. This applies to all urban sizes that define urban space. From city scale, buildings, squares, parks, and streets to details of these elements, such as street furniture, garden trees, and more. The design of these interconnected elements, which make up the urban environment of a city, is called urban design.

As the world moved to think of more sustainable cities, architecture and urban design had a share, to be more sustainable. In a good choice of the principles of sustainable urban design, which vary and differ according to theories of sustainable urban design, the researcher and in a previous study of her doctoral thesis summarized seven major themes of high urban design that can be applied to universal cities, it relied on the detailed principles of the six sustainable urban design theories (Green urbanism, Sustainable urbanism, New urbanism, Cittaslow, Smart city, Islamic city). These principles that were applied to the Libyan city of Zawiya in a comprehensive study for the design of the city's development strategy, namely: Regulate the urban structure, Sustainable movement network, Environmental compatibility, High quality of buildings, Smart people, Good governance policies, Economic policy.
Among these principles and vocabulary of sustainable urban design, "walkability" is an excellent abbreviation for good urban design.

\section{OBJECTIVE}

Determine the problems and obstacles facing parties involved in moving within Zawiya city. Solve problems from the traffic congestion and a lack of city vibrancy to the absence of social contact and obesity epidemic.

Focusing on change the priorities of the streets design of the city, from the existing situation that is focusing only on vehicles, to an integrated process that takes into account the needs of pedestrians and passengers, drivers, cyclists, and motorists alike

\section{Sustainable neighborhoods}

Some cities around the world are conceiving of alternative models for those prevalent in urban development, which are dependent on economic growth. These models focus on building environmentally friendly cities. It embraced innovative thinking in the field of urban planning, urban design, and urban technology, to highlight some of the evolutionary solutions that change the world's vision of cities. 
Ideas defended how to use resources today without compromising their availability in the future, clarifying a definition of sustainability and its rules. She emphasized that cities must meet the needs of their residents to be sustainable. However, the definition of urban areas is still a long way off, as cities have many different characteristics. Zones can be classified as urban based on the population exceeding a certain number (population thresholds), number of people per square meter (population density), or the presence of basic urban services such as water and electricity networks and educational and health facilities [1].

In urban design, it is determined by the importance of the presence of the elements that define urban design, such as: architecture, landscape, streets, public places, and transport systems. In fact, a highquality urban design is sustainable. Places are carefully designed and attentive to details of elements such as street furniture, building facades, building responses, green and blue spaces, light and shadow. One of the conditions for successful urban design to be more quality is respect for all vital elements involved in the design. Which gives the city its activity and its attributes, such as pedestrians, vehicles and trees. Attention to each individual item and providing its needs without harming others.

Spatial design and planning are carried out by providing accessible transportation systems. The urban form refers to crowded, small, and mixed-use spaces, in an integrated framework of public transportation, environmental policy and management.

\section{Identifying walkability}

The street is not for movement only, but also to support land uses, located along its extent, including spaces for cafes seats, and places of social networking, and children's playgrounds, and public places, as it contribute to the economic success of the institutions located there.

When designing a good design of the street, and attention to pedestrians and the possibility of pedestrian access easily to the facilities, and provide areas of shade, it gives a feeling of security in different times of the day, whether at night or during the day, it makes the walk enjoyable and safety, and reduces the driving rates, and improves health. As well, designing streets to accommodate all types of transportation, and using landscaping and irrigation works related thoughtfully, and improve the level of comfort for pedestrians, it can achieve a significant reduction in carbon emissions and the impact of rising temperatures inside the cities and water consumption.

South worth defined the (Walkability) as an extension or space in which the built environment is supportive and encouraging for walking, by providing comfort and safety for pedestrians and connecting people with various arrival goals during a reasonable period of time and effort as well as providing visual attractions during the trip within this network [2].

Walkability is a measure of the effectiveness of urban form and community design. "Walkability" means promoting walking, biking to replace the use of cars for shopping, going to school and public places. Moreover, "Walkability" enhances design sustainability, enhancing physical fitness and fighting obesity [3].

It is possible to assess the ability to walk on several levels: at the street level, at the neighborhood unit level, or at the community level. To achieve high levels of walkability, Ogra and Ndebele define 6Ds to development walkability: Destination Accessibility, Diversity, Density, Design, Distance to Transit, and Parking, Street connectivity and low vehicle speeds [1]

\section{METHODOLOGY}

The paper is based on the standard approach to assess ability of walkability, which is the analysis of traditional urban geo-data related to accessibility (Linda Dörrzapf). This method is based on spatial data analysis. Datasets are aggregated on various spatial scales and include urban functions, street network, landuse classification, width of roads, and population.

\section{EVALUATING THE WALKABILITY IN URBAN DESIGN OF ZAWIYA}

Zawiya City is a small city located at the Mediterranean Sea, 40km west of the capital Tripoli. The city lacks the elements of sustainable urban design. The researcher has defined the urban landscape of the city: The urban determinants lack communication between people and the surrounding environment. The streets are for cars only, the gardens are almost nonexistent, the buildings are not consistent, and the hot sun penetrates everywhere.

To assess the walkability of Zawiya, the most important street in the city, Jamal Street, was chosen as a model to be studied, analysis and design. It is a historical street that has been defined the features of the city and developed around it, through which people crossed every day for decades to reach Tripoli or Sabratha [5].

The streets of Zawiya city classified, multi-use zones of moderate levels of pedestrian activities, where surrounding buildings usually consists of three to six floors. Jamal Street classifies, a medium to low priority, depending on the different uses of land on both sides of the street: from Zawiya University, and the university hospital, to the Institute of Teachers, and historical buildings (the Security Directorate and the old high school). Jamal Street crosses the city from east to west with $6 \mathrm{~km}$ long, from the eastern bridge at the entrance 
to the city from the direction of Tripoli city, to the western bridge at the entrance to the city from direction of Sabratha city and Tunis [5].

The street under study was divided into certain lengths, determined by the street itself. Where it is observed that the street is self-divided, at the intersections of the convergence with the main vertical streets on it, which are installed by the roundabouts placed at the intersections.

These divisions contributed to making each part of the street length has specific features in terms of density, road width, land use classification, and even design details.

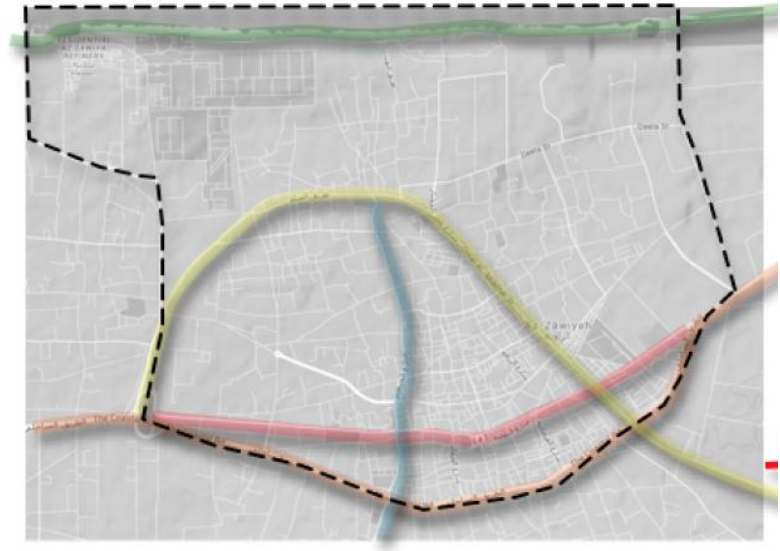

Fig-1: Map shows main streets in Zawia city, Jamal Street (red line)

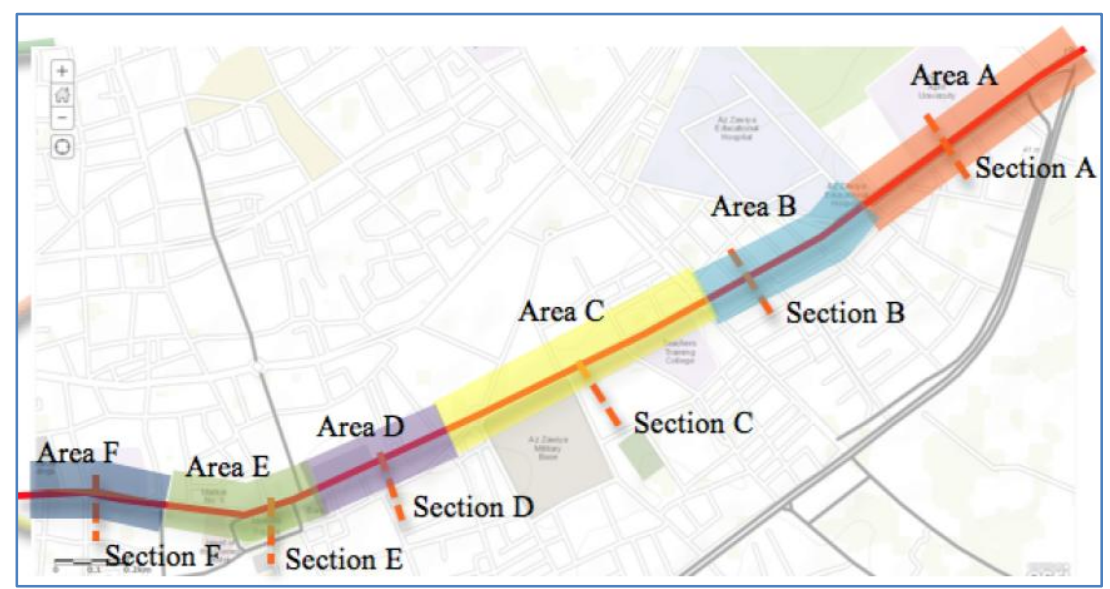

Fig-2: Six sections in different areas in Jamal Street (A, B, C, D, E, F) according to width, intensity and land uses

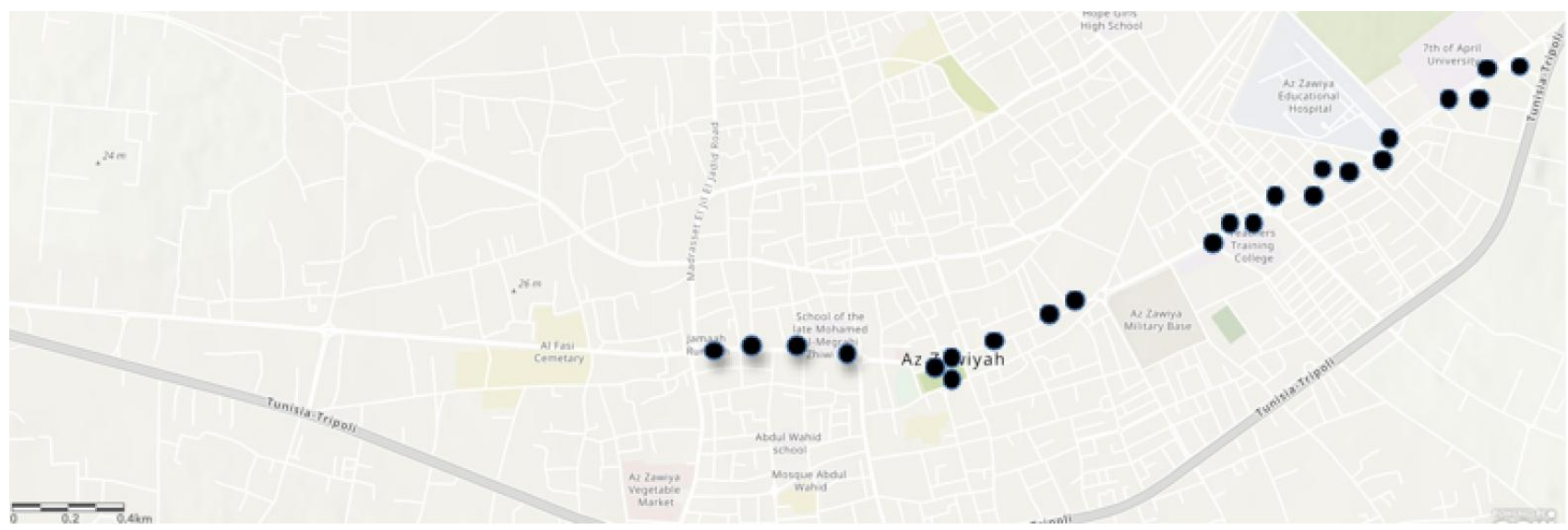

Fig-1: The picture shows the locations of the pedestrian gathering, and it can be seen that there is a separation between areas 2,4 , which the use of land in area 3 changes to administrative areas 


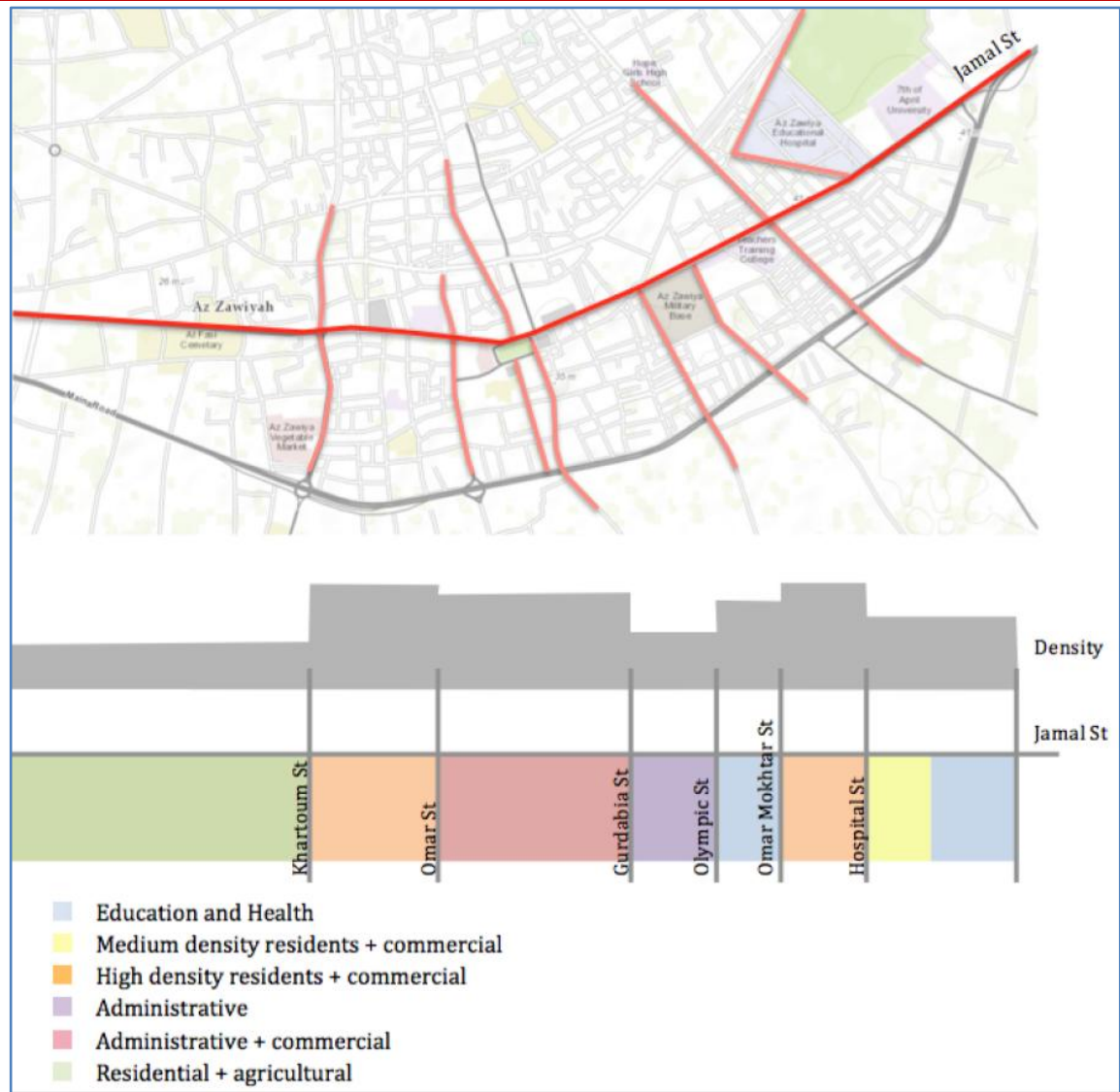

Fig-2: The picture shows the locations of the pedestrian gathering, and it can be seen that there is a separation between areas 2,4 , which the use of land in area 3 changes to administrative areas

Table-1: An analysis of the walkability factors of Jamal Street

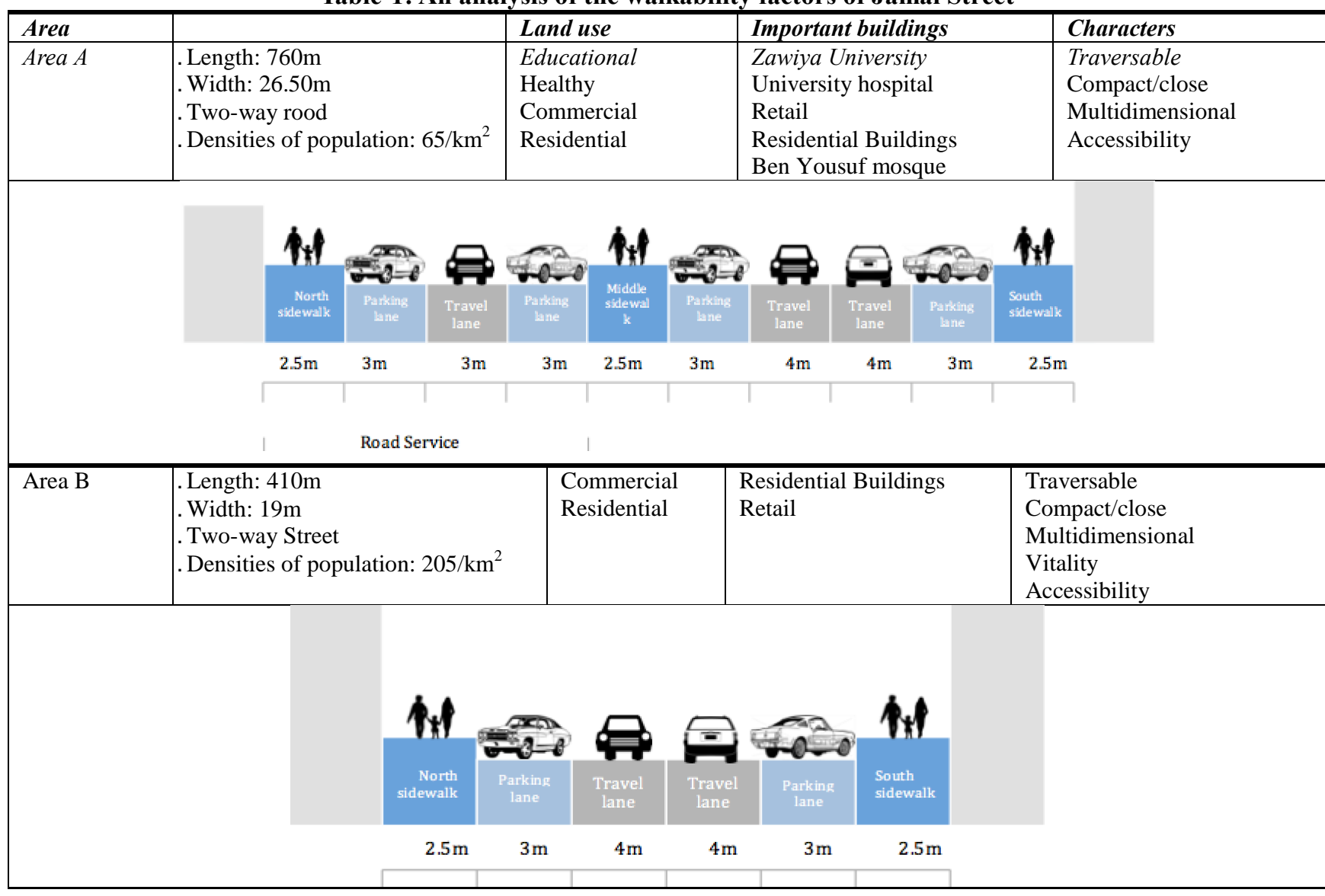




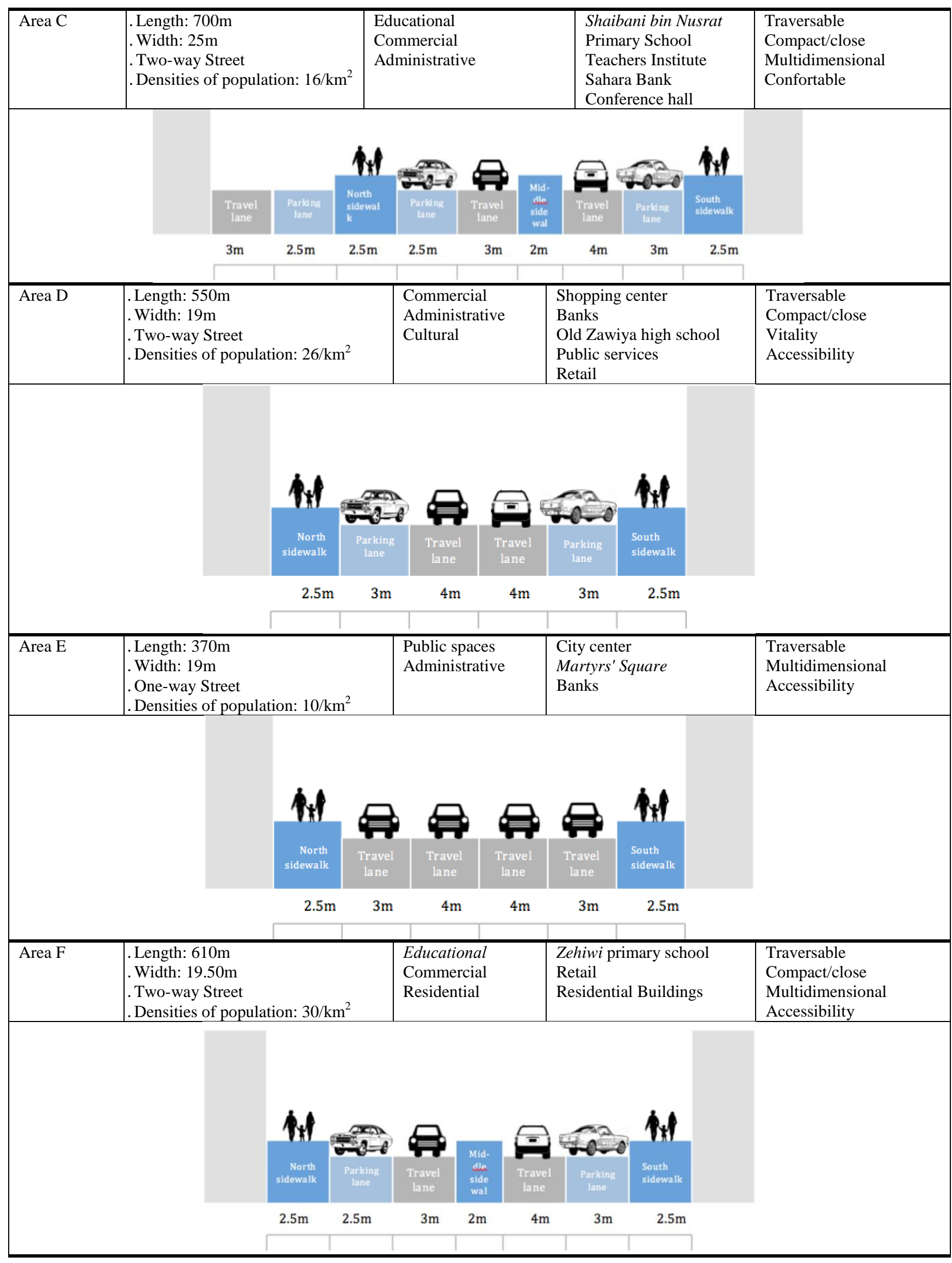




\section{Feedback}

Among the streets that have been observed, this region has the most walking trips. This does not include walking trips undertaken by the residents of the region, but also from outside the region, often due to the presence of Al-Zawiya University and the university hospital. Students contributed to the increase in the density of pedestrians, on weekdays and in the morning hours, while the number of pedestrians has decreased significantly at the end of the week, especially on Friday, as the holy weekend in the Libyan Islamic cities.

Most of the buildings in the area are for residential use and public services, and a high percentage of them have retail outlets at ground level, which creates a very mixed-use environment. The ground floor of the buildings connects to the pavement in a traditional urban block way, and creates direct connecting with the pedestrians, and this provides a lively and safe environment for walking, as shops and cafes generate activities that allow direct and indirect interaction with the pedestrians. Not only for movement, but through store window displays that enhance the appeal of walking.

There are some points define the case of walkability of Jamal Street of Zawiya, on account of:

- The pattern of the street is changing almost every meter.

- Buildings on the same pattern, on the edge of the street, developed randomly, without being restricted by construction regulations, they have different height, with many attempts to increase height of buildings by residents.

- Although the street is commercial, Parking is not available for the region; both for the population or a pedestrian, what makes people parked cars next to the sidewalk, that Ineligible.

- There are no organized traffic lights, or white pedestrian lines.

- There is no good design for pedestrian confluence, and there are no attractions for places

- The street lacks elements of good urban design

- There are no design considerations for vehicles, bicycles, and pedestrians

\section{Design Considerations}

Achieving a balance between the needs of all mobility, as a priority arrangement: Pedestrians, Public transport, Cyclists, Motorists as follow:

\section{Pedestrians}

Pedestrians are the first priority for the design of the street, which should be safe and comfortable for all ages and physical abilities. Pedestrians have special needs as a key part of the design of the walkability:

- As a places protection and parasols, and trees to cope with high temperatures in the summer. Estimate the pedestrian crossing times when street intersections.

- Development special specifications for the disabled, the elderly, such as: places to use wheelchairs, motorized vehicles, and the possibility of their use of pedestrian paths. Bearing in mind the slow movement rather quickly.

- Soft non-slip lanes, footpaths and barrier-free.

- Provide safety and security for women and children to move easily through the streets.

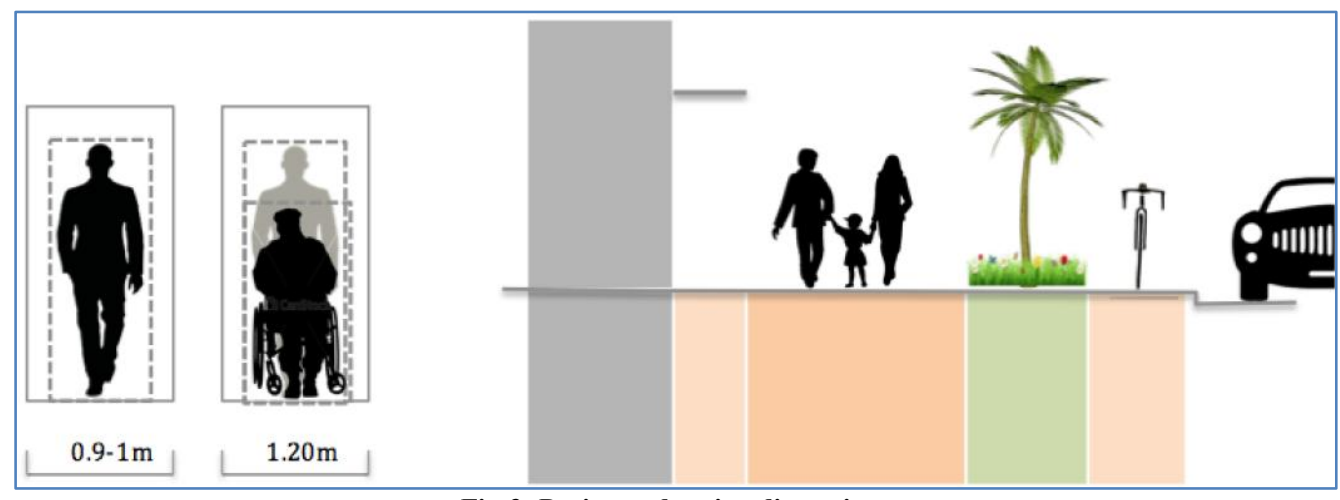

Fig-3: Design pedestrian dimensions 


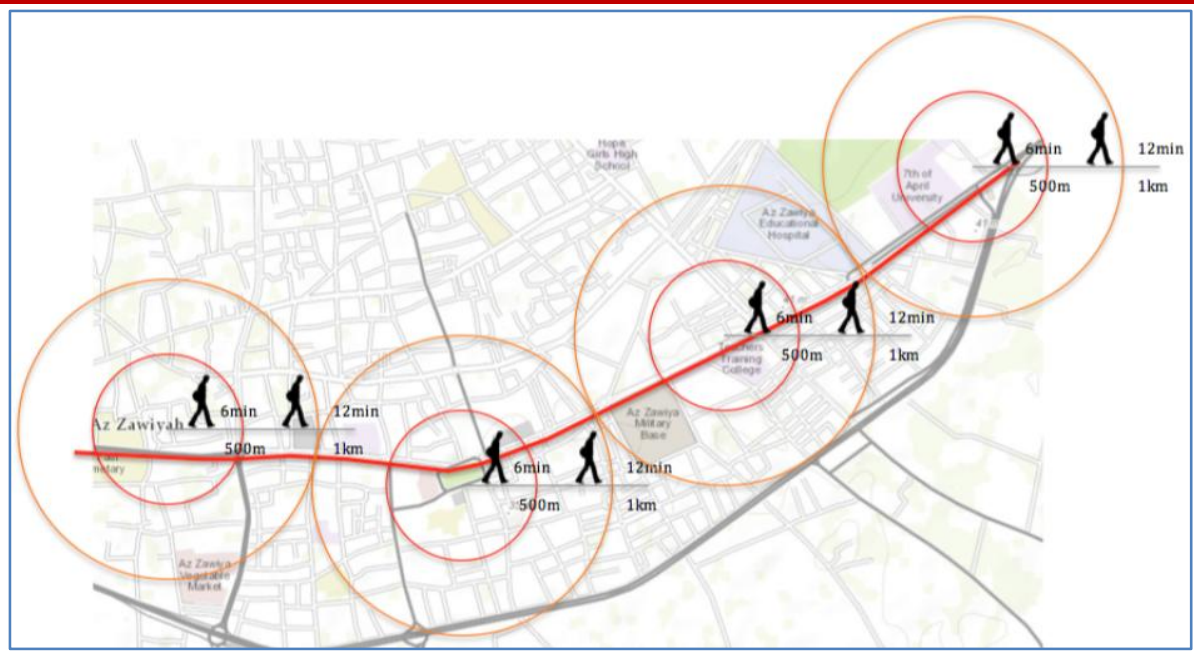

Fig-4: Calculating walking distances of Jamal Street, in minutes and meters

\section{Cyclists}

Bicycles drivers are vulnerable road users, and should take into account their own safety during the design. They are also considered to be among the most effective of the streets of users. Cycling provides a means of transport in effectiveness, and environmentally friendly, and healthy. Rate ranges bike speed wind between 15 and 20 kilometers per hour, but it vary according to the age and activity of the passenger and desires.

It can provide customized bicycle facilities within the area of pedestrians in the form of paths for bicycles, or within the allotted cars Street as lanes for bicycles, or side lanes.

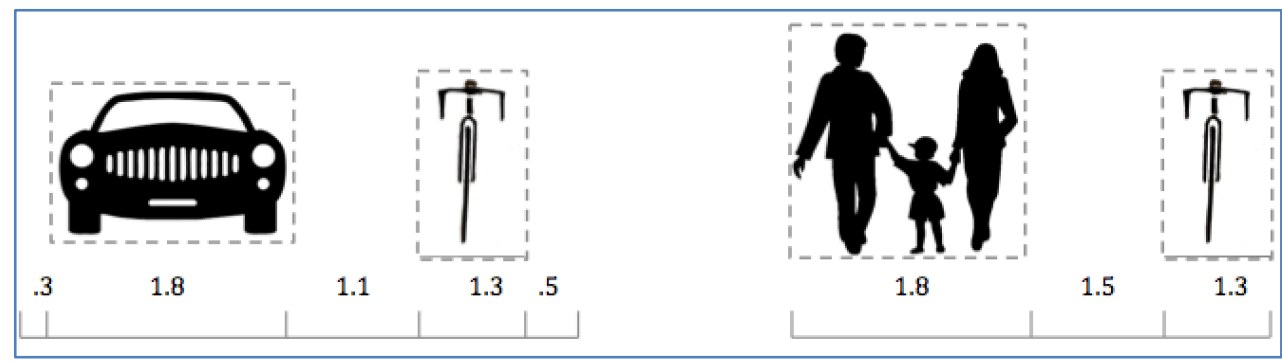

Fig-5: Design Bicycle dimensions in meters

\section{Motorists}

Accommodate traffic cars is very important to achieve continuous growth. Design speed is, selected speed, to determine the design of the various dimensions of the street, and sometimes to determine the timing of the traffic signals.

The minimum speed is one of the main characteristics of a world-class city take into account the safety and freedom of walking is $15 \mathrm{~km}$ per hour.
When the street design, taking into account, emergency service vehicles, cars and freight service.

\section{Public transport}

Auto transport users are among the most effective of the streets of users. The basic needs of the users of public transport safety, security and comfort in waiting areas. And be able to cross the street effective and easy to get to the positions allocated to public transport vehicles.

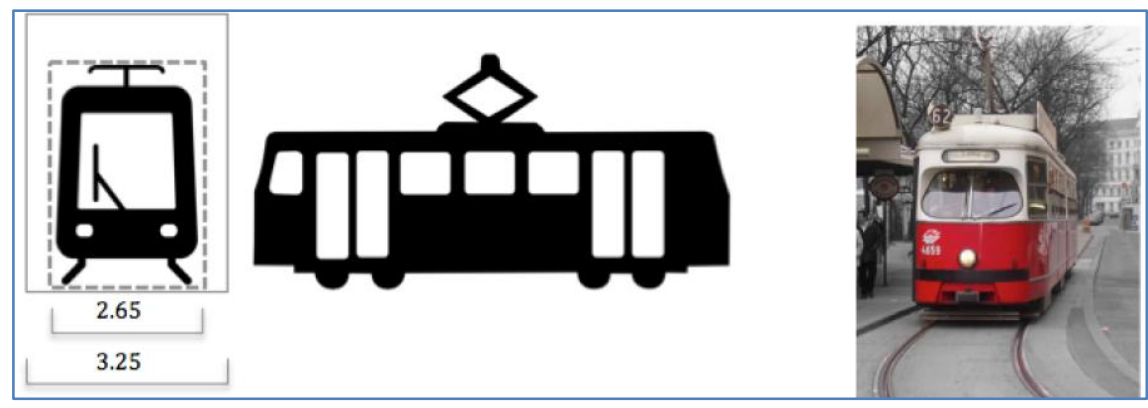

Fig-6: Simple spatial dimensions for railroad cars (tram) 


\section{CONCLUSION}

It was noted that by analyzing the city streets, it contains some good design basics for streets, along with some other factors that make the city sustainable.

This paper discusses the study of spatial data for Jamal Street in the city of Zawiya, and it is considered a partial environmental design guide for the city streets. In order to make progress towards a more comprehensive understanding of the viability of traffic, one must also focus on subjective factors such as perceptions and individual emotions.

This guide will help officials and residents to make the city a social place, more comfortable and safer for all users, as we will move to the next stage, which is street design.

\section{REFFERENCE}

1. United Nations. (2014). World Urbanization Prospects: the 2014 revision, highlights .

2. Forsyth, A., \& Southworth, M. (2008). Cities afoot-Pedestrians, walkability and urban design.

3. UN-Habitat. (2013). Streets as public spaces and drivers of urban prosperity. United Nations Human Settlements Programme. Kenya : UN-Habitat.

4. AlAhrash, A. (2010). Spatial variation in the implementation of housing plans of Zawiya city during 1980-2008. Libya Academy, Geography , Tripoli.

5. Elbasha, H. (2015). Origination and historical development of Zawia city . Okan University, Architecture, Istanbul. 